\title{
Rescue of recombinant peste des petits ruminants virus: creation of a GFP-expressing virus and application in rapid virus neutralization test
}

Qianqian $\mathrm{Hu}^{1,2}$, Weiye Chen ${ }^{1,2^{*}}$, Kehe Huang ${ }^{1}$, Michael D Baron ${ }^{3}$ and Zhigao Bu ${ }^{1,2^{*}}$

\begin{abstract}
Peste des petits ruminants virus (PPRV) causes high mortality in goats and sheep and the disease has shown a greatly increased geographic distribution over the last 15 years. It is responsible for serious socioeconomic problems in some of the poorest developing countries. The ability to create recombinant PPRV would provide a useful tool for investigating the biology of the virus and the pathology of disease, as well as for developing new vaccines and diagnostic methods. Here we report the first successful rescue of recombinant PPRV from a full-length cDNA clone of the virus genome. Successful recovery of PPRV was achieved by using a RNA polymerase II promoter to drive transcription of the full-length virus antigenome. We have used this technique to construct a virus expressing a tracer protein (green fluorescent protein, GFP). The recombinant virus replicated as well as the parental virus and could stably express GFP during at least 10 passages. The newly established reverse genetics system for PPRV provides a novel method for constructing a vaccine using PPRV as a vector, and will also prove valuable for fundamental research on the biology of the virus. We found that our recombinant virus allowed more rapid and higher throughput assessment of PPRV neutralization antibody titer via the virus neutralization test (VNT) compared with the traditional method.
\end{abstract}

\section{Introduction}

Peste des petits ruminants (PPR) is a highly contagious disease of domestic and wild small ruminants caused by peste des petits ruminants virus (PPRV); it is responsible for serious socioeconomic problems in some of the poorest developing countries [1-3]. PPR is a notifiable disease listed by the World Organisation for Animal Health (OIE). PPR was first reported in the Ivory Coast in 1942, and later found in the Middle and Near East, southwest and central Asia [4-6], and recently in China [7]. PPRV, which is a member of the genus Morbillivirus belonging to the family Paramyxoviridae [8], is a linear,

\footnotetext{
* Correspondence: chenweiye1980@yahoo.com.cn; zgbu@yahoo.com ${ }^{1}$ College of Veterinary Medicine, Nanjing Agricultural University, Nanjing 210095, People's Republic of China

${ }^{2}$ The Key Laboratory of Zoonoses of Chinese Academy of Agricultural Sciences, Key Laboratory of Veterinary Public Health of Ministry of Agriculture, State Key Laboratory of Veterinary Biotechnology, Harbin Veterinary Research Institute of Chinese Academy of Agricultural Sciences, Harbin 150001, People's Republic of China

Full list of author information is available at the end of the article
}

non-segmented, single stranded, negative-sense RNA virus with a genome length of 15948 bp. The PPRV genome encodes six structural proteins (nucleocapsid $(\mathrm{N})$, phosphoprotein (P), matrix (M), fusion (F), hemagglutinin $(\mathrm{H})$, and polymerase $(\mathrm{L}))$, and two nonstructural proteins $(\mathrm{C}$ and $\mathrm{V})$, which are in the order of $3^{\prime}-\mathrm{N}-\mathrm{P} / \mathrm{C} / \mathrm{V}-\mathrm{M}-\mathrm{F}-\mathrm{H}-\mathrm{L}-5^{\prime}$ on the genome [9-12]. PPRV vaccine strain (Nigeria 75/1, PPRV/N75/1) has been widely used as a safe and efficacious live vaccine to control PPR infections [13].

Several studies have indicated that recombinant paramyxoviruses are effective and genetically stable vectors with many advantages [14-16] due to their relatively simple reverse genetic systems. However, a reverse genetic system for PPRV has so far not been possible, despite effort in several laboratories, although a rescue system for rinderpest virus (RPV), which is evolutionary closest to PPRV, has been known since 1997 [17], and a

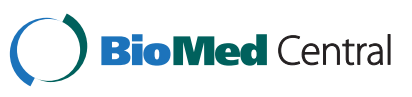


PPRV mini-genome rescue system was described in 2007 [18].

Green fluorescent protein (GFP) is a useful tracer protein to observe and optimize virus rescue efficiency, and to study the characteristics of rescued viruses. Recombinant viruses expressing GFP could also be utilized to genetically mark vaccines to allow serological differentiation between animals that have been vaccinated against PPR and those recovering from natural infection $[16,19]$ or to improve the virus neutralization test (VNT) [2023]. In this study, we developed a system for recovering recombinant PPRV and introduced the GFP open reading frame into a recombinant form of PPRV/N75/1 to create a marked recombinant PPRV which we have used initially to improve PPRV VNT assays for use in studies of immune responses to different vaccines.

\section{Materials and methods}

\section{Cells and viruses}

PPRV vaccine strain (Nigeria 75/1, PPRV/N75/1) was obtained from the China Institute of Veterinary Drug Control, Beijing, China. Vero cells (The American Type Culture Collection, ATCC: CCL-81) were cultured in Dulbecco's modified Eagle's medium (DMEM) (Gibco, Carlsbad, CA, USA) containing 10\% fetal bovine serum (FBS) (Gibco). Vero cells expressing the canine form of the general morbillivirus receptor, signaling lymphocyte activation molecule (SLAM), (VDS cells) were the kind gift of Dr P Duprex, Queen's University Belfast, UK and were maintained in DMEM/10\% FBS/0.1 mg/mL Zeocin. PPRV/N75/1 or rescued recombinant PPRV were propagated and titrated in Vero cells cultured in DMEM containing 2\% FBS.

\section{Serum samples}

Ten goats (nos. 1-10) and 10 sheep (nos. 11-20) were vaccinated twice with $2 \times 10^{5} 50 \%$ tissue culture infective dose $\left(\mathrm{TCID}_{50}\right)$ of recombinant capripoxvirus (rCPV) expressing PPRV glycoprotein H (rCPV-PPRVH) [24] with a three-weeks interval. Serum samples were collected two weeks following the second vaccination. Eleven additional goats (nos. 21-31) were vaccinated with $10^{7}$ TCID $_{50}$ PPRV/N75/1, and serum samples were collected four weeks post-vaccination. Serum samples were collected from each test animal before vaccination to act as negative controls.

\section{Plasmid construction}

PPRV/N75/1 was propagated in Vero cells, and RNA from infected cells isolated. The entire viral genome was amplified by RT-PCR using high-fidelity $P f x$ DNA polymerase (Invitrogen, Carlsbad, CA, USA) in four overlapping sections (F1 to F4), which were assembled into a full-length cDNA clone (Figure 1a). The complete cDNA of the genome of the virus stock used was fully sequenced and confirmed [GenBank: HQ197753]. A number of minor differences between the sequence determined for this stock of the PPRV/N75/1 vaccine and that previously published for PPRV/N75/1 [GenBank: X74443] were noted, but it is not possible to tell if these are due to mistakes in the earlier sequence or to changes to the vaccine seed stock over time. The hammerhead ribozyme sequence (HamRz) and hepatitis delta virus ribozyme sequence ( $\mathrm{HdvRz}$ ) were introduced at the $5^{\prime}$ and $3^{\prime}$ ends of the antigenomic sequence, respectively, as previously described by Inoue et al. [25,26]. The assembled HamRz-(full-length genomic cDNA)$\mathrm{HdvRz}$ was then cloned into the pCI vector (Promega, Madison, WI, USA) under the control of the CMV promoter. The resulting plasmid was named pN75/1 (Figure 1a).

New gene fragments were then inserted into pN75/1 between nt 3405 and 3406 of the PPRV/N75/1 sequence (i.e. at the start of the $M$ gene) as illustrated in Figure 1b: a 32 nt gene start (GS) sequence (5'-aggagcaagggcaactgagcttcacagacaag-3'), a Not I restriction site, a Pme I restriction site, a 66 nt gene end (GE) sequence (5' -cacatcctataatcaacatctcatactcggttgaaaacatcctctcaatcaggctattacaaaaa-3') and a CTT intergenic trinucleotide. In brief, the genome construction was carried out as follows: DNA fragment Fa (ending at the GS of the M gene with a Not I site introduced at 3' end) and $\mathrm{Fb}$ (starting from the GE of the P gene with Not I and Pme I sites introduced at the $5^{\prime}$ end) were PCR-amplified from pN75/1 and ligated together to get DNA Fab, then DNA F1 used in the original construction of $\mathrm{pN} 75 / 1$ was replaced with DNA Fab to give plasmid pN75/1-insertion (Figure 1b). The net result was equal to insertion of the five genetic elements above into pN75/1 between nt 3405 and 3406 of the PPRV/N75/1 genome cDNA sequence.

Finally, we inserted the open reading frame (ORF) for GFP into pN75/1-insertion. The GFP ORF was PCRamplified from pIRES2-EGFP (Clontech, Mountain View, CA, USA) using PrimeSTAR HS DNA polymerase (Takara, Shiga, Japan) with primers $5^{\prime}$-tctgcggccgc gccgccaccatggtgagcaagggcgag- $3^{\prime}$ (the Not I site is underlined and the Kozak sequence is in bold) and $5^{\prime}$-ctc gtttaaacttacttgtacagctcgtc-3' (the Pme $I$ site is underlined). The amplified product was ligated into EcoR $V$ cut pBluescript II $\mathrm{KS}(+)$, removed from that plasmid by digestion with Not I and Pme I and cloned into pN75/1insertion, cut with the same enzymes, to produce plasmid pN75/1-GFP (Figure 1c).

In addition, the ORFs of the $\mathrm{N}, \mathrm{P}$ and $\mathrm{L}$ genes were amplified from pN75/1 and inserted into pCAGGS to construct helper plasmids. The resultant plasmids were named $\mathrm{pCA}-\mathrm{N}, \mathrm{pCA}-\mathrm{P}$ and $\mathrm{pCA}-\mathrm{L}$, respectively. All 


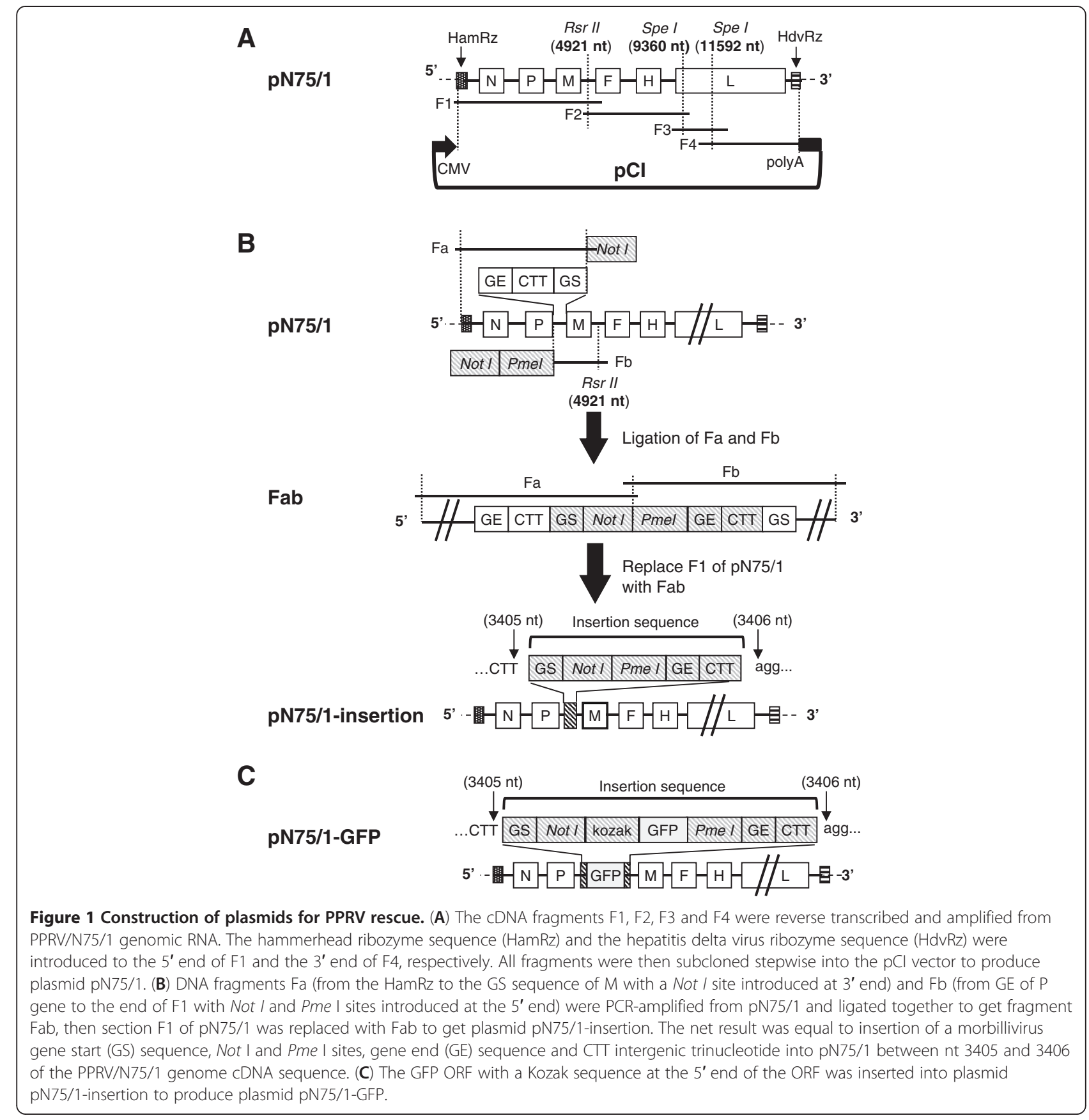

primer sequences used in this study are available from the corresponding author upon request.

\section{Transfection of Vero cells and rescue of recombinant viruses from cloned cDNA}

To rescue the recombinant PPRV or PPRV/GFP, 90\% confluent Vero or VDS cells in one well of a 6-well plate were transfected with the plasmids pCA-N $(2 \mu \mathrm{g})$, pCA$\mathrm{P}(1 \mu \mathrm{g})$ and pCA-L $(1 \mu \mathrm{g})$ together with $4 \mu \mathrm{g}$ of pN75/ 1-GFP. Lipofectamine 2000 (Invitrogen) or TransIt-LT1
(Mirus Biologicals) were used for transfections following the manufacturers' instructions. After 7-9 days of incubation at $37^{\circ} \mathrm{C}$, the cells and supernatants were collected and freeze-thawed twice and then passaged in fresh cells to propagate the rescued virus. Supernatants from cytopathic effect-positive wells were used to propagate viral stocks in Vero or VDS cells. The complete genomic sequences of the rescued viruses were confirmed by sequencing. The rescued viruses were named rPPRV/N75/ 1 and $\mathrm{rPPRV} / \mathrm{GFP}$. 


\section{Growth curves}

Vero cells were grown to $70 \%$ confluence in 6-well plates and infected with 0.1 multiplicity of infection (MOI) of virus for $1 \mathrm{~h}$. The inoculum was removed and the cells were washed twice with medium, then $2 \mathrm{~mL}$ of medium were added to each well. Cells with medium were stored at $-70^{\circ} \mathrm{C}$ each day from 3 to 8 days postinfection, and were freeze-thawed twice before titration. The $\mathrm{TCID}_{50}$ of released virus was quantitated by established methods [27].

\section{Immunofluorescence assays}

Vero-SLAM cells grown in 12-well plates on glass coverslips were infected with PPRV/N75/1 or $\mathrm{rPPRV} / \mathrm{GFP}$ at a MOI of 0.05 and incubated for $2 \mathrm{~d}$. Cells were fixed with 3\% paraformaldehyde in PBS and stained with monoclonal antibody recognizing PPRV $\mathrm{H}$ followed by AlexaFluor 568-labelled anti-mouse IgG (Invitrogen). Cells were stained with DAPI for 5 min before mounting to stain the nuclei of all cells. Images were taken by sequential scanning at each wavelength on a Leica confocal microscope. Mock-infected cells were used as controls. For live cell imaging, Vero cells were infected at a MOI of 0.1 and imaged at the indicated time post infection using an inverted fluorescence microscope (Zeiss, Oberkochen, Germany).

\section{Western blotting}

Vero cells were infected with PPRV/N75/1 or rPPRV/ GFP at a MOI of 0.1 and incubated until the cytopathic effect (CPE) involved $60-80 \%$ of cells. The cell extracts were then analyzed by SDS-PAGE and blotted on a nitrocellulose membrane. The membrane was incubated with mouse anti-GFP monoclonal antibody (Sigma) as a primary antibody, and with peroxidase-conjugated goat anti-mouse IgG (Sigma) as a secondary antibody. Immunostained proteins were visualized using 3,3' -diaminobenzidine reagent. Mock-infected Vero cells were used as controls.

\section{Analysis of GFP fluorescence}

Vero cells grown in 6-well plates were infected with rPPRV/GFP at a MOI of 0.1. The cells together with their medium were freeze-thawed twice when the CPE reached $100 \%$, and $200 \mu \mathrm{L}$ of this broken cell preparation was added to $100 \mu \mathrm{L}$ of cell-lysis buffer $(0.15 \mathrm{M}$ Tris-Cl, $\mathrm{pH}$ 8.0, 1.5\% Triton X-100). After incubating for $15 \mathrm{~min}, 100 \mu \mathrm{L}$ aliquots of cell lysate were transferred to wells of a 96-well white plate (Corning, Lowell, MA, USA). Mock-infected cells were used as controls. The GFP fluorescence of each well was read on a Microplate Fluorescence Reader (Bio-Tek, Winooski, VT, USA). The excitation peak was set at $485 \mathrm{~nm}$, the emission peak at $528 \mathrm{~nm}$, and the sensitivity at 50. The relative fluorescence units (RFU) were calculated as: [(fluorescence of the test well) - (fluorescence of the control well)].

\section{Virus neutralization tests}

Titrating of PPRV-neutralizing antibody (VNA) in serum samples were performed in quadruplicate in 96-well plates as previously described [24] following OIE recommendations [28]. All serum samples were inactivated by heating at $56^{\circ} \mathrm{C}$ for $30 \mathrm{~min}$ before testing. The inactivated sera were diluted five-fold in triplicate, and then serially diluted two-fold for VNA titration. PPRV/N75/1 or $\mathrm{rPPRV} / \mathrm{GFP}\left(100 \mathrm{TCID}_{50}\right.$ in $100 \mu \mathrm{L}$ cell culture medium) was mixed with $100 \mu \mathrm{L}$ of diluted serum in a 96-well plate and incubated at $37^{\circ} \mathrm{C}$ for $1 \mathrm{~h}$. Vero cells $(50 \mu \mathrm{L})$ were added to each well and the plates were incubated at $37^{\circ} \mathrm{C}$. The CPE was recorded at day 14 for PPRV/N75/1 as described previously [28]. A titer $\geq 10$ was considered positive.

\section{Statistical analysis}

The statistical analyses of the comparison between the results of assays using PPRV/75/1 and rPPRV/GFP were carried out using a paired $t$ test as calculated using the GraphPad Prism program. A P value $<0.05$ was considered significant.

\section{Results}

\section{Rescue of rPPRV and GFP expression of rPPRV/GFP in vitro}

Previous attempts to rescue PPRV from full-length copies of the genome had used T7 RNA polymerase-driven transcription of the virus antigenome, since this method has been successful with all other morbilliviruses, indeed most viruses of the order Mononegavirales, rescued to date. We hypothesised that the PPRV rescue may not have worked because of some sequence element in the PPRV genome (e.g. cryptic transcription termination signals) that were preventing full genome synthesis. We therefore attempted the recovery of recombinant PPRV using an RNA pol II promoter to drive transcription, and ribozymes at both ends of the PPRV sequence to ensure that the final transcript had exact viral termini, as has been shown to be effective for rabies virus rescue [25]. We therefore constructed the PPRV genome plasmid as described in Methods (Figure 1) as well as appropriate helper plasmids expressing the $\mathrm{N}, \mathrm{P}$ and $\mathrm{L}$ proteins of PPRV, and rPPRV/N75/1 and rPPRV/GFP were rescued successfully on Vero cells. Because rPPRV/ GFP is much easier than $\mathrm{rPPRV} / \mathrm{N} 75 / 1$ for evaluating or optimizing the reverse genetic system, rPPRV/GFP was used in all following experiments. Optimum ratios of plasmids were determined based on the frequency of GFP-positive cells after transfection, and we were able to 

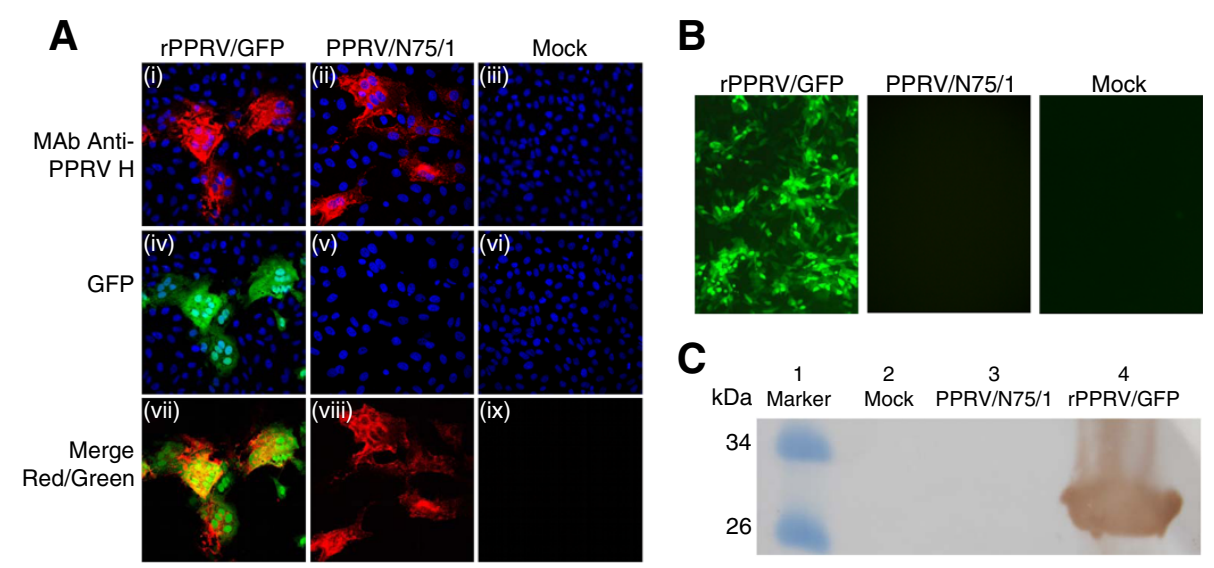

Figure 2 Replication of and GFP expression by rPPRV/GFP in Vero cells. (A) Cells infected with either rPPRV/GFP (i, iv, vii) or PPRV/N75/1 (ii, $\mathrm{V}$, viii), or mock infected (iii, vi, ix), were fixed and labeled for the presence of PPRV glycoprotein $\mathrm{H}$ (red); GFP was detected by its natural fluorescence. Cells were counterstained with DAPI to show the nuclei. (B) GFP expression in live, PPRV/N75/1- and rPPRV/GFP-infected Vero cells was observed by direct observation of unfixed cells 2 days post infection. (C) Lysates of Vero cells that had been infected with PPRV/N75/1 or rPPRV/GFP were analyzed by Western blotting using mouse anti-GFP monoclonal antibody. Mock-infected Vero cells were used as a controls.

recover recombinant PPRV/GFP in $50 \%-80 \%$ of transfected wells using the final method as described.

To confirm rPPRV/GFP replication and normal viral protein expression as well as GFP expression, infected VDS cells were immunostained with anti-PPRV $\mathrm{H}$ MAb as described in Methods. Immunofluorescence microscopy showed clear labeling of cells infected with either PPRV/N75/1 or rPPRV/GFP (Figure 2a), while only cells infected with rPPRV/GFP showed the green fluorescence expected of GFP expression (Figure 2a). The GFP expression was strong enough to be seen even in early stages of infection, as shown by the appearance of cells at the borders of infection foci which were clearly green but had not yet expressed detectable amounts of the viral $\mathrm{H}$ protein. Infected cells could easily be seen by live cell imaging (Figure 2b). The expression of GFP was further confirmed by Western blot analysis, which showed that a protein of the appropriate size was detected with anti-GFP antibody in lysates of rPPRV/GFP-infected cells (Figure 2c, lane 4), while no band was detected with mock- or PPRV/N75/1infected cells (Figure 2c, lanes 2 and 3).

\section{Virus growth and stability in vitro}

To determine whether the rescue procedure or exogenous gene insertion affected the replicative ability of our recombinant virus, growth curves of rPPRV/GFP and PPRV/N75/1 in infected Vero cells were determined. The results (Figure 3a) show that there was no discernible difference in growth rate or maximum titre between the two viruses. To determine whether repeated passage of rPPRV/GFP in Vero cells affected replication and expression, rPPRV/GFP was propagated in Vero cells for 10 passages, and samples of culture medium plus cells collected every second passage; these samples were
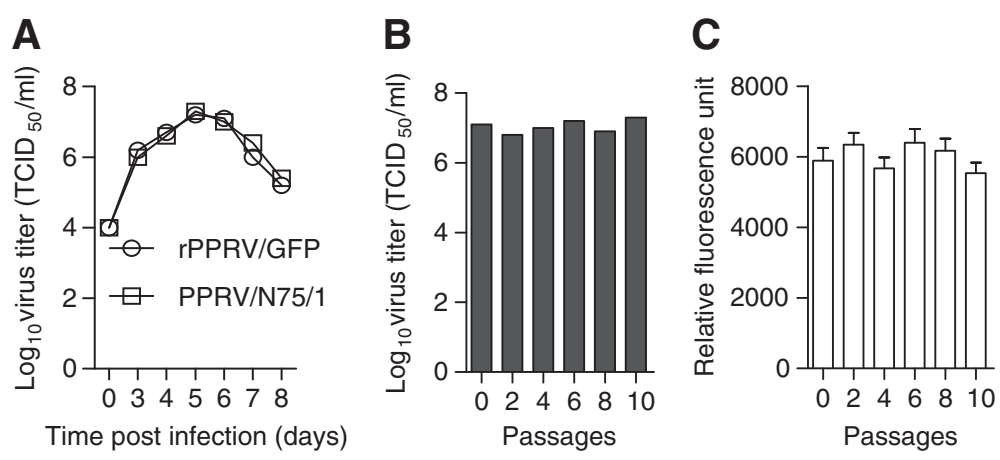

Figure 3 Growth and stability of rPPRV/GFP in vitro. (A) Vero cells were infected with PPRV/N75/1 or rPPRV/GFP, and the amount of virus in the cultures was measured at various times from 0 to $8 \mathrm{~d}$ post-infection. (B) rPPRV/GFP was passaged in Vero cells every 6-7 d, and culture medium and cells of each passage was freeze-thawed twice and used for virus titration (B) and to analyze GFP expression by quantitating relative fluorescence (C). Data are expressed as the mean \pm standard deviation (SD). 
assayed for total (cell associated and medium) virus titre and for GFP expression. The results show that the virus titer (Figure 3b) and GFP expression (Figure 3c) from different passages changed only slightly.

\section{PPR virus neutralization test using rPPRV/GFP}

GFP expression from our rPPRV/GFP is easily observed in live cells by fluorescence microscopy. We took advantage of this to help us observe virus growth during the carrying out of VNTs, growth that is normally detected by the observation of cytopathic effect (CPE). Serum from a vaccinated sheep (no. 31) was tested in a VNT assay using rPPRV/GFP or PPRV/N75/1. GFP fluorescence (Figure 4a (i-iv)) and CPE (Figure 4a (ix-xii)) of rPPRV/GFP or CPE of PPRV/N75/1 (Figure 4a (xiii-xvi)) was observed at 4, 6, 10 and $14 \mathrm{~d}$ post-infection in wells where virus was incubated with 320 -fold diluted serum. The GFP fluorescence could be observed as early as day
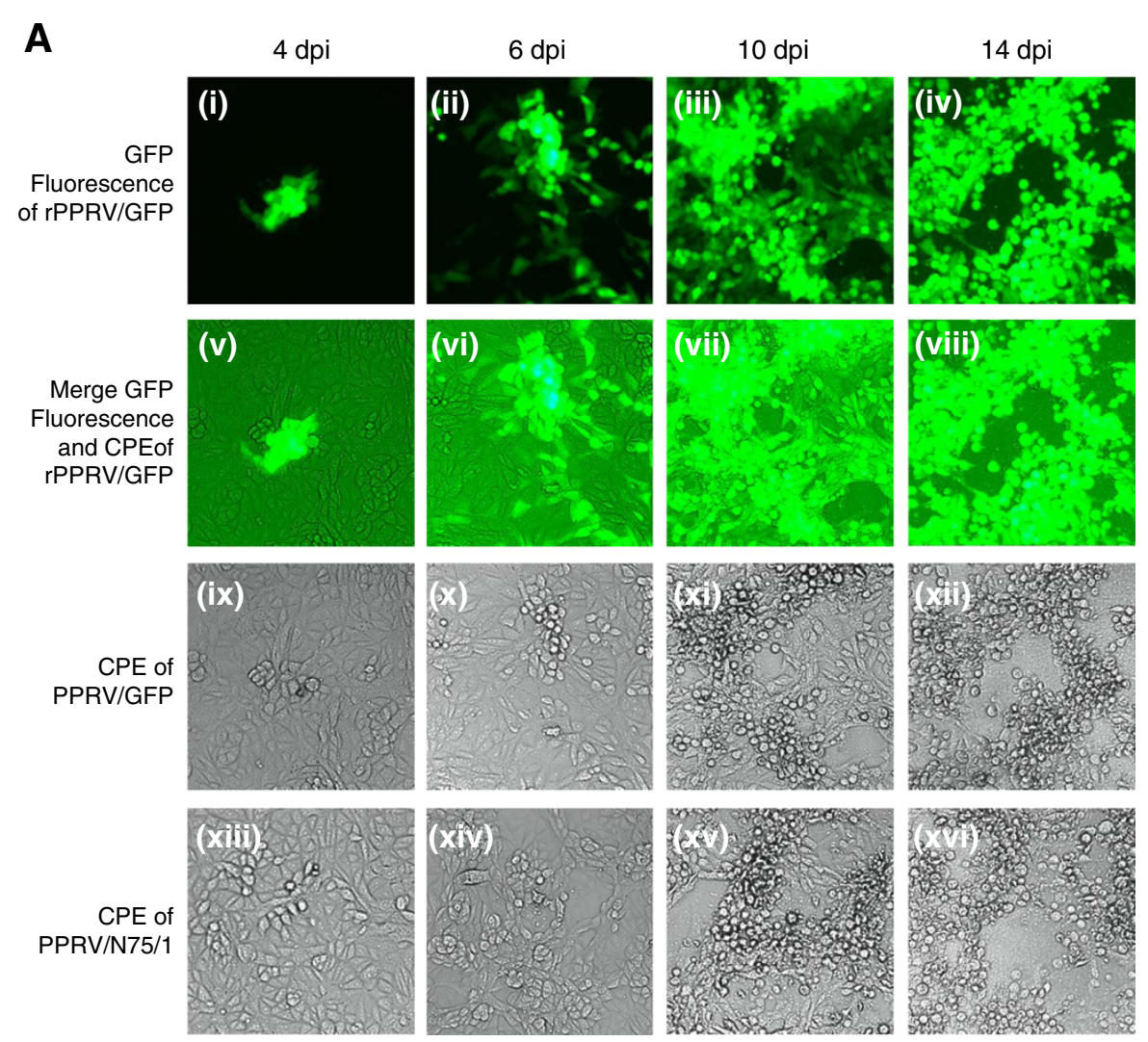

B
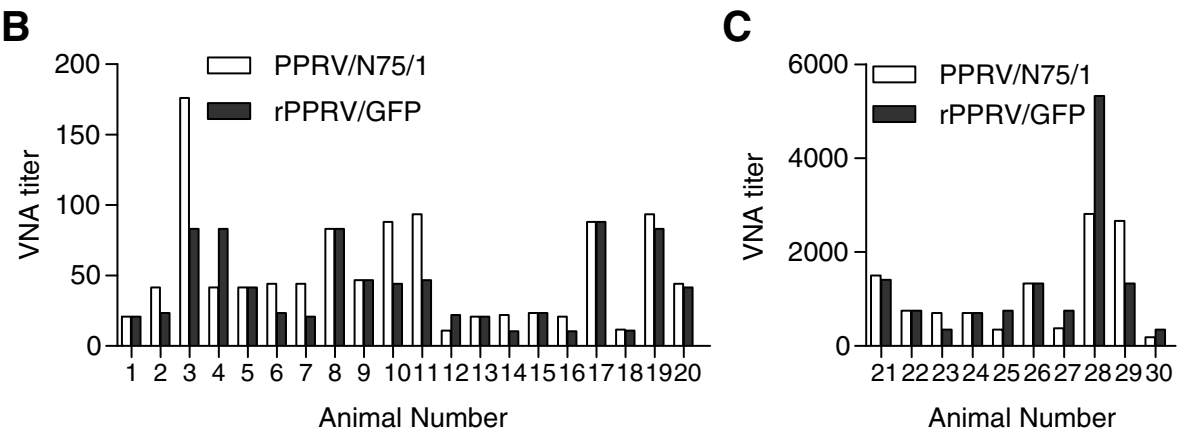

Figure 4 Comparison of PPR virus neutralization test using rPPRV/GFP and PPRV/N75/1. (A) Detection of virus replication during VNT assay. Serum from a goat (no. 31) previously vaccinated with $10^{7} \mathrm{TCID}_{50}$ PPRV/N75/1 was tested using either rPPRV/GFP or PPRV/N75/1. The GFP fluorescence ((i) to (iv)) and CPE ((ix) to (xii)) of rPPRV/GFP or the CPE of PPRV/N75/1 ((xiii) to (xvi)) after treatment with diluted serum (320-fold) were observed at 4, 6, 10 and 14 d post-infection. (B) Twenty PPRV-positive sera from rCPV-PPRVH vaccinated goats (nos. 1-10) or sheep (nos. 11-20) were assayed for PPR VNA titer using PPRV/N75/1 and rPPRV/GFP. (C) Ten positive sera from PPRV/N75/1 vaccinated goats (nos. 21-30) were assayed in the same manner as above. The results from assays with the two viruses were compared using a paired $t$ test, and no significant difference seen. 
4 (Figure $4 \mathrm{a}(\mathrm{i})$ ), and was very clear after day 6 (Figure 4a (ii-iv)), whereas the PPRV/GFP (Figure 4a (xixii)) and PPRV/N75/1 CPE (Figure 4a (xv-xvi)) were only clearly observed from day 10 . Therefore, the rPPRV/GFP VNT results were available at least four days earlier than if using PPRV/N75/1.

In order to verify that the two methods gave the same titre, 20 PPRV-positive sera from rCPV-PPRVH vaccinated goats (nos. 1-10) or sheep (nos. 11-20) were assayed for PPR VNA titer using either PPRV/N75/1 or $\mathrm{rPPRV} / \mathrm{GFP}$. As shown in Figure 4b, there was no statistically significant difference $(P>0.05$, paired $t$-test) between the VNA titer results using the two viral strains. Ten additional positive serum samples from PPRV/N75/1-vaccinated goats (nos. 21-30), which have much higher VNA titres, were assayed in the same manner, and with same results $(P>0.05$, Figure $4 \mathrm{c})$. The VNA titer of 30 (negative) serum samples collected from 30 test animals (nos. 1-30) before vaccination were all lower than 5 (data not shown) using either rPPRV/GFP or PPRV/N75/1.

\section{Discussion}

Here, a full PPRV reverse genetics system has been established for the first time. Since the establishment of a RPV reverse genetics system more than a decade ago [17], several groups have attempted to establish a PPRV reverse genetics system $[18,29]$. However, the attempts have been unsuccessful to date (personal communication). We have now successfully rescued a recombinant PPRV expressing GFP. Rescue efficiencies were acceptable, but not high, and rescue efficiency may have to be improved to enable recovery of PPRV with more extensive or deleterious mutations, either to use PPRV as a novel vaccine vector or for fundamental research.

Our results showed that neither rescue conditions nor insertion of an additional gene affected recombinant virus replication and passage stability in Vero cells. This is in contrast to the findings with similar recombinant viruses made using the vaccine strain of RPV $[19,30]$, where the insertion of an extra gene between the virus $\mathrm{P}$ and $\mathrm{M}$ genes led to a reduction in growth rate. GFP expression was high, suggesting potential use of a PPRV reverse genetics system to construct recombinant multivalent vaccines by replacing the GFP ORF with the coding sequence for an immunogenic antigen from another virus, or the creation of tagged viruses for use in fundamental research on the growth and spread of PPRV in its hosts, as has been recently carried out with measles virus [31,32]. A version of rPPRV/GFP could be used as a marker vaccine, especially if GFP is expressed as a membrane-anchored protein, which was necessary with RPV-based constructs to elicit a serum antibody response to the GFP $[16,19]$.

PPRV/N75/1 replicates relatively slowly in Vero cells, with 14 days typically required for observation of CPE in
VNT assays where low initial MOIs are used. CPE during early infection (1-6 d post-infection) can be very difficult to observe. In addition, complex serum components may cause CPE-like cell death at dilutions below 20-fold even when the sera are heat-inactivated at $56^{\circ} \mathrm{C}$. When the VNA titer is between 5 and 20 , it is difficult to judge whether cell death has been caused by virus or by the serum itself, which may lead to falsenegative results and underestimation of VNA titre. rPPRV/GFP could easily solve the two above-mentioned problems. First, virus growth can be observed with the help of GFP fluorescence as early as four days post infection in these assays, and VNT results could be determined with confidence as early as six days post infection. Thus, eight days could be saved compared with traditional methods using PPRV/N75/1. Secondly, viral CPE could be distinguished easily from CPE-like cell death caused by serum with the help of GFP fluorescence, even though only a limited number of cells are infected by rPPRV/GFP. There is also the potential to use machine scanning of wells of 96-weel plates in a fluorescence plate reader to automate scoring of VNT assays, allowing higher throughput of samples, an important consideration where large scale trials of vaccines or tests for vaccine effectiveness are taking place.

\section{Competing interests}

ZB and WC are inventors on a pending patent application for the reverse genetics system of PPRV and rPPRV/GFP. Other authors declare no financial or non-financial competing interests in the publication of this work.

\section{Acknowledgements}

This work was supported by National Natural Science Foundation of China (30901082), by National High Technology Research and Development Program of China (863) (2011AA10A208), and by A Project Funded by the Priority Academic Program Development of Jiangsu Higher Education Institutions (PAPD). The laboratory of MDB is supported by the UK Biotechnology and Biological Sciences Research Council (BBSRC).

\section{Author details}

${ }^{1}$ College of Veterinary Medicine, Nanjing Agricultural University, Nanjing 210095, People's Republic of China. ${ }^{2}$ The Key Laboratory of Zoonoses of Chinese Academy of Agricultural Sciences, Key Laboratory of Veterinary Public Health of Ministry of Agriculture, State Key Laboratory of Veterinary Biotechnology, Harbin Veterinary Research Institute of Chinese Academy of Agricultural Sciences, Harbin 150001, People's Republic of China. ${ }^{3}$ Institute for Animal Health, Pirbright Laboratory, Pirbright, Surrey GU24 ONF, United Kingdom.

\section{Authors' contributions}

$\mathrm{QH}$ and WC carried out all the experiments except confocal microscopy and contributed to the drafting of the manuscript; $\mathrm{KH}$ contributed to the cloning and sequencing of PPRV genome RNA; MDB contributed to the construction of the genome plasmid, carried out the confocal microscopy and contributed to the drafting of the manuscript; ZB designed the whole study, provided general supervision and prepared the manuscript. All authors have read and approved the submitted manuscript.

Received: 22 March 2012 Accepted: 2 June 2012

Published: 2 June 2012 
References

1. Lefevre PC, Diallo A: Peste des petits ruminants. Rev Sci Tech 1990, 9:935-981

2. Khan $H$, Siddique M, Abubakar M, Arshad M, Hussain M: Prevalence and distribution of peste des petits ruminants virus infection in small ruminants. Small Ruminant Res 2008, 79:152-157.

3. Sen A, Saravanan P, Balamurugan V, Rajak KK, Sudhakar SB, Bhanuprakash V, Parida S, Singh RK: Vaccines against peste des petits ruminants virus. Expert Rev Vaccines 2010, 9:785-796

4. El Hag Ali B, Taylor WP: Isolation of peste des petits ruminants virus from the Sudan. Res Vet Sci 1984, 36:1-4.

5. Amjad H, ul Qamar I, Forsyth M, Barrett T, Rossiter PB: Peste des petits ruminants in goats in Pakistan. Vet Rec 1996, 139:118-119.

6. Kwiatek O, Minet C, Grillet C, Hurard C, Carlsson E, Karimov B, Albina E, Diallo A, Libeau G: Peste des Petits Ruminants (PPR) Outbreak in Tajikistan. J Comp Pathol 2007, 136:111-119.

7. Wang Z, Bao J, Wu X, Liu Y, Li L, Liu C, Suo L, Xie Z, Zhao W, Zhang W, Yang N, Li J, Wang S, Wang J: Peste des petits ruminants virus in Tibet, China. Emerg Infect Dis 2009, 15:299-301.

8. Gibbs EP, Taylor WP, Lawman MJ, Bryant J: Classification of peste des petits ruminants virus as the fourth member of the genus Morbillivirus. Intervirology 1979, 11:268-274.

9. Muthuchelvan D, Sanyal A, Sreenivasa BP, Saravanan P, Dhar P, Singh RP, Singh RK, Bandyopadhyay SK: Analysis of the matrix protein gene sequence of the Asian lineage of peste-des-petits ruminants vaccine virus. Vet Microbiol 2006, 113:83-87.

10. Abubakar M, Khan HA, Arshed MJ, Hussain M, Ali Q: Peste des petits ruminants (PPR): Disease appraisal with global and Pakistan perspective. Small Ruminant Res 2011, 96:1-10

11. Renukaradhya GJ, Sinnathamby G, Seth S, Rajasekhar M, Shaila MS: Mapping of B-cell epitopic sites and delineation of functional domains on the hemagglutinin-neuraminidase protein of peste des petits ruminants virus. Virus Res 2002, 90:171-185.

12. Bailey D, Banyard A, Dash P, Ozkul A, Barrett T: Full genome sequence of peste des petits ruminants virus, a member of the Morbillivirus genus. Virus Res 2005, 110:119-124.

13. Diallo A, Taylor WP, Lefevre PC, Provost A: Attenuation of a strain of rinderpest virus: potential homologous live vaccine. Rev Elev Med Vet Pays Trop 1989, 42:311-319. in French.

14. Ge J, Deng G, Wen Z, Tian G, Wang Y, Shi J, Wang X, Li Y, Hu S, Jiang Y, Yang C, Yu K, Bu Z, Chen H: Newcastle disease virus-based live attenuated vaccine completely protects chickens and mice from lethal challenge of homologous and heterologous $\mathrm{H} 5 \mathrm{~N} 1$ avian influenza viruses. J Virol 2007, 81:150-158.

15. Ge J, Wang X, Tao L, Wen Z, Feng N, Yang S, Xia X, Yang C, Chen H, Bu Z: Newcastle disease virus-vectored rabies vaccine is safe, highly immunogenic, and provides long-lasting protection in dogs and cats. $J$ Virol 2011, 85:8241-8252

16. Walsh EP, Baron MD, Rennie LF, Monaghan P, Anderson J, Barrett T: Recombinant rinderpest vaccines expressing membrane-anchored proteins as genetic markers: evidence of exclusion of marker protein from the virus envelope. J Virol 2000, 74:10165-10175.

17. Baron MD, Barrett $\mathrm{T}$ : Rescue of rinderpest virus from cloned cDNA. J Virol 1997, 71:1265-1271.

18. Bailey D, Chard LS, Dash P, Barrett T, Banyard AC: Reverse genetics for peste-des-petits-ruminants virus (PPRV): promoter and protein specificities. Virus Res 2007, 126:250-255.

19. Walsh EP, Baron MD, Anderson J, Barrett T: Development of a genetically marked recombinant rinderpest vaccine expressing green fluorescent protein. J Gen Virol 2000, 81:709-718.

20. Biacchesi S, Skiadopoulos MH, Yang L, Murphy BR, Collins PL, Buchholz UJ: Rapid human metapneumovirus microneutralization assay based on green fluorescent protein expression. J Virol Methods 2005, 128:192-197.

21. Johnson MC, Damon IK, Karem KL: A rapid, high-throughput vaccinia virus neutralization assay for testing smallpox vaccine efficacy based on detection of green fluorescent protein. J Virol Methods 2008, 150:14-20.

22. Yoshii K, Ikawa A, Chiba Y, Omori Y, Maeda J, Murata R, Kariwa H, Takashima I: Establishment of a neutralization test involving reporter geneexpressing virus-like particles of tick-borne encephalitis virus. J Virol Methods 2009, 161:173-176.
23. Rimmelzwaan GF, Verburgh RJ, Nieuwkoop NJ, Bestebroer TM, Fouchier RA, Osterhaus AD: Use of GFP-expressing influenza viruses for the detection of influenza virus A/H5N1 neutralizing antibodies. Vaccine 2011, 29:34243430.

24. Chen W, Hu S, Qu L, Hu Q, Zhang Q, Zhi H, Huang K, Bu Z: A goat poxvirus-vectored peste-des-petits-ruminants vaccine induces longlasting neutralization antibody to high levels in goats and sheep. Vaccine 2010, 28:4742-4750.

25. Inoue K, Shoji Y, Kurane I, lijima T, Sakai T, Morimoto K: An improved method for recovering rabies virus from cloned cDNA. J Virol Methods 2003, 107:229-236.

26. Tao L, Ge J, Wang X, Zhai H, Hua T, Zhao B, Kong D, Yang C, Chen H, Bu Z Molecular basis of neurovirulence of flury rabies virus vaccine strains: importance of the polymerase and the glycoprotein R333Q mutation. $J$ Virol 2010, 84:8926-8936.

27. Reed $\sqcup$, Muench $H$ : A simple method of estimating fifty percent endpoints. Am J Hygiene 1938, 27:493-497.

28. OIE: Manual of Diagnostic Tests \& Vaccines for Terrestrial Animals. 6th edition. Paris:; 2008.

29. Zhai JJ, Dou YX, Zhang HR, Mao L, Meng XL, Luo XN, Cai XP: Construction and sequencing of full-length CDNA of peste des petits ruminants virus. Bing Du Xue Bao 2010, 26:315-321. in Chinese.

30. Baron MD, Foster-Cuevas M, Baron J, Barrett T: Expression in cattle of epitopes of a heterologous virus using a recombinant rinderpest virus. J Gen Virol 1999, 80:2031-2039.

31. de Vries RD, Lemon K, Ludlow M, McQuaid S, Yuksel S, van Amerongen G, Rennick LJ, Rima BK, Osterhaus AD, de Swart RL, Duprex WP: In vivo tropism of attenuated and pathogenic measles virus expressing green fluorescent protein in macaques. J Virol 2010, 84:4714-4724.

32. de Swart RL, Ludlow $M$, de Witte L, Yanagi $Y$, van Amerongen G, McQuaid S, Yuksel S, Geijtenbeek TB, Duprex WP, Osterhaus AD: Predominant infection of CD150+ lymphocytes and dendritic cells during measles virus infection of macaques. PLoS Pathog 2007, 3:e178.

doi:10.1186/1297-9716-43-48

Cite this article as: Hu et al:: Rescue of recombinant peste des petits ruminants virus: creation of a GFP-expressing virus and application in rapid virus neutralization test. Veterinary Research 2012 43:48.

\section{Submit your next manuscript to BioMed Central and take full advantage of:}

- Convenient online submission

- Thorough peer review

- No space constraints or color figure charges

- Immediate publication on acceptance

- Inclusion in PubMed, CAS, Scopus and Google Scholar

- Research which is freely available for redistribution 